\title{
Optical image correlation using acoustooptic and charge-coupled devices
}

\author{
Demetri Psaltis
}

\begin{abstract}
An optical processing method is presented which allows the correlation of two images to be computed in real time with a 1-D acoustooptic spatial light modulator as the input device. Two-dimensional processing is accomplished by a combination of spatial and temporal integration. The time-integrating processing is performed by a CCD detector that is operated as an optically addressed correlator.
\end{abstract}

\section{Introduction}

Optical image processors normally require at the input stage a real-time 2-D spatial light modulator $(\mathrm{SLM})^{1}$ which creates a suitable transparency corresponding to the input image. The relatively immature state of development of 2-D SLMs is a major factor that has prevented the realization of the huge potential of optical image processors. The limitations presently imposed by the SLMs can be overcome either by further developments in device technology or alternatively by modifying the optical system architecture. Several architectural designs have been proposed for this purpose. ${ }^{2-4}$ For example, if the optical processor can be implemented with noncoherent illumination the requirements on the optical quality of the SLM can be relaxed, and a self-luminous display (such as a CRT) could be used as the input SLM. In a recent publication by the author ${ }^{3}$ a $2-D$ signal processing technique has been presented that does not require the use of real-time 2-D SLMs. This is accomplished by entering the 2-D input data into the optical processor one line at a time using a 1-D acoustooptic SLM. ${ }^{5}$ Each line is filtered spatially by the optical system. The data are processed in the second dimension by integrating the response due to consecutive lines on a time-integrating detector array. Acoustooptic technology is highly developed and consequently high quality acoustooptic SLMs can be fabricated. The use of acoustooptic devices (AOD) rather than 2-D SLMs in the optical processor can lead to improvements in accuracy, speed, and practicality (size, weight, power requirements, cost). In

\footnotetext{
The author is with California Institute of Technology, Department of Electrical Engineering, Pasadena, California 91125.

Received 7 August 1981.

0003-6935/82/030491-05\$01.00/0.

(C) 1982 Optical Society of America.
}

this paper we describe the application of the timeand-space integrating processing technique to the implementation of an image correlator. Image correlation is a useful signal processing operation in pattern recognition, image restoration and enhancement, and video compression and is also the most powerful operation that can be implemented by an optical computer. Therefore, image correlation is probably the most significant 2-D signal processing operation that can be implemented with the time-and-space integrating method. In the following section we describe the operation of the optical image correlator, and in Sec. III we discuss the unique characteristics of this architecture.

\section{Description of the Processor}

The time-and-space image correlating architecture is shown in Fig. 1. We will demonstrate that this system computes the cross-correlation $g(\hat{x}, \hat{y})$ of two complex functions $f(x, y)$ and $h(x, y)$ :

$$
g(\hat{x}, \hat{y})=\iint f(x, y) h^{*}(x+\hat{x}, y+\hat{y}) d x d y .
$$

The operation of the correlator in Fig. 1 can be summarized as follows: the optical system is a multichannel 1-D correlator which consecutively correlates each row of the function $f(x, y)$ with all rows of the function $h(x, y)$. The AOD is used to enter the rows of $f(x, y)$ sequentially into the optical system. The 1-D correlations are summed appropriately by operating the CCD detector in the shift-and-add mode. ${ }^{6}$ The key components of the system are the AOD and the CCD detectors.

The AOD consists of a piezoelectric transducer bonded onto an acoustooptic crystal. We denote the voltage applied to the piezoelectric transducer by $s(t)$. The signal $s(t)$ has the form $s(t)=\alpha(t) \cos \left[w_{0} t+\phi(t)\right]$, where the complex envelope $a(t)=\alpha(t) \exp [j \phi(t)]$ is the modulating signal and $w_{0} / 2 \pi$ is the center frequency of the AOD. The device is illuminated by a collimated light beam incident at the Bragg angle $\theta_{B}$, where ${ }^{7}$ 

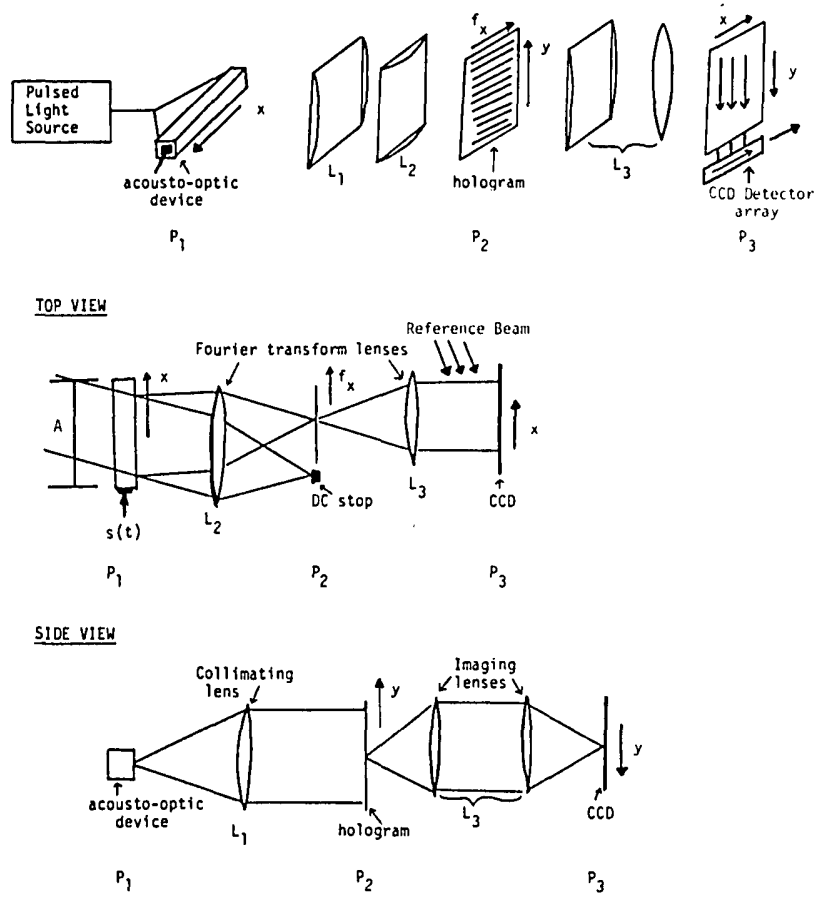

Fig. 1. Image correlator architecture.

$$
\sin \theta_{B}=\frac{\lambda w_{0}}{4 \pi v} .
$$

$\lambda$ is the wavelength of light in the acoustooptic crystal, and $v$ is the speed of sound in the crystal. The acoustic wave that is induced by the voltage $s(t)$ causes a portion of the incident light to be diffracted. In the Bragg regime essentially all the diffracted energy is concentrated in the first order. For relatively weak modulation levels the amplitude of the diffracted light $E\left(x^{\prime}, t\right)$ is given by ${ }^{8}$

$$
\begin{aligned}
E\left(x^{\prime}, t\right) \cong & c_{1} \operatorname{rect}\left(\frac{x^{\prime}-A / 2}{A}\right) \alpha\left(t-\frac{x}{v}\right) \\
& \times \exp \left[j \phi\left(t-\frac{x}{v}\right)\right] \exp \left[j w_{0}\left(t-\frac{x}{v}\right)\right] \exp \left(j \frac{2 \pi \sin \theta_{B} x}{\lambda}\right) \\
= & c_{1} \operatorname{rect}\left(\frac{x^{\prime}-A / 2}{A}\right) a\left(t-\frac{x}{v}\right) \\
& \times \exp \left(j \omega_{0} t\right) \exp \left(-j \frac{2 \pi \sin \theta_{B} x^{\prime}}{\lambda}\right) .
\end{aligned}
$$

In Eq. (3) $c_{1}$ is a constant, $x^{\prime}$ is along the direction of the acoustic wave propagation, and $A$ is the aperture of the AOD. Thus, the amplitude of the diffracted light is modulated by the complex signal $a\left(t-x^{\prime} / v\right)$ over the spatial window $A$, Doppler shifted by the traveling acoustic wave by $\omega_{0} / 2 \pi$, and deflected around the Bragg angle $\theta_{B}$. For the rest of our discussion we will not carry the deflection term $\exp \left[-j\left(2 \pi \sin \theta_{B} x^{\prime}\right) / \lambda\right]$ in the equations since it does not affect the operation of the system we will describe. In Eq. (3) the origin of the $x^{\prime}$ coordinate is assumed to be at the piezoelectric transducer, and $x^{\prime}$ increases away from the transducer. In our analysis it is more convenient to use the coordinate transformation $x=-x^{\prime}+A$. In the $x$-coordinate system the origin is at the far end of the acoustooptic crystal, and $x$ increases toward the transducer. Equation (3) can be rewritten as follows:

$$
E(x, t)=c_{1} \operatorname{rect}\left(\frac{x-A / 2}{A}\right) a\left(t+\frac{x}{v}-\frac{A}{v}\right) \exp \left(j w_{0} t\right) .
$$

We shall use Eq. (4) in the rest of our discussion.

The CCD image sensor consists of a matrix of photosensitive sites where incident photons are converted to electronic carriers which are trapped locally in the depletion region of a MOS junction. 9 The photogenerated charge packets can be transferred to adjacent pixels along one of the dimensions of the array by applying a clock waveform to the device. When the device is used as an image sensor, each charge packet is shifted continuously along the array after a single exposure to light until it reaches the edge of the device where an output CCD stage transfers the signal to the output pin. In our system the CCD array is exposed periodically. After each exposure the charge that is accumulated in each site is shifted by only 1 pixel. The photogenerated charge due to the most recent exposure is simply added to the charge that is already stored in each pixel. In Fig. 1 the spatial dimension along the shifting direction of the CCD is denoted by $\hat{y}$. Let the intensity of the illuminating light at the location $\hat{y}$ during the $n$th exposure be denoted by $I(n, \hat{y})$. The charge generated at the pixel located at $\hat{y}$ during the $n$th exposure is proportional to $I(n, \hat{y})$. After $N$ exposures $(N \geq n)$, the charge $I(n, \hat{y})$ shifts $N-n$ pixels or by a distance $(N-n) \Delta y$ in the $\hat{y}$ direction. $\Delta y$ is the pixel separation. The charge that is accumulated in the CCD as a function of pixel position $\hat{y}$ after $N$ exposures is given by

$$
Q(\hat{y})=\sum_{n=1}^{N} I[n, \hat{y}+(n-N) \Delta y],
$$

for $\hat{y}=0$ to $\hat{y}=M \Delta y$, where $M$ is the number of pixels in the $\mathrm{CCD}$ and $I(n, \hat{y})=0$ for all $\hat{y} \leq 0$. The maximum number of exposures $N$ for which the addition in Eq. (3) can be performed is equal to $M$. Bromley, and Monahan et al. ${ }^{6}$ have used a CCD detector array in this mode of operation to perform a variety of 1-D linear operations. In our system we use the CCD detector to construct an array of parallel 1-D correlators.

In most image recognition applications the input scene and the filter functions are real. For this reason and to present the principles of operation of the system more clearly, we will restrict our analysis initially to real functions $f$ and $h$. For completeness we will describe the operation of the system with complex signals $f$ and $h$ at the end of this section. The 1-D Fourier transform of a transparency with amplitude transmittance $h(x, y)$ is formed with an astigmatic lens system which transforms in the $x$ direction and images along $y .10$ A hologram of the resulting light amplitude distribution is formed by recording its interference with a plane wave reference on photographic film or any other suitable device. The amplitude transmittance of the developed hologram is proportional to ${ }^{10}$ 


$$
\begin{aligned}
&\left|B \exp \left(-j 2 \pi \sin \theta_{H} F f_{x}\right)+H\left(f_{x}, y\right)\right|^{2} \\
&=B^{2}+\left|H\left(f_{x}, y\right)\right|^{2} \\
&+B H^{*}\left(f_{x}, y\right) \exp \left(-j 2 \pi \sin \theta_{H} F f_{x}\right) \\
&+B H\left(f_{x}, y\right) \exp \left(+j 2 \pi \sin \theta_{H} F f_{x}\right)
\end{aligned}
$$

where

$$
H\left(f_{x}, y\right)=\int h(x, y) \exp \left(-j 2 \pi f_{x} x d x,\right.
$$

$\theta_{H}$ is the angle of incidence on the hologram of the reference beam, $B$ is its amplitude, $F$ is the focal length of the lens in the $x$ direction, and $f_{x}$ is the spatial frequency variable which is linearly related to the spatial variable $\xi$ in the plane of the hologram $\left(f_{x}=\xi /(\lambda F)\right)$. In Eq. (6) only the term containing $H^{*}$ contributes to the correlation, and the remaining terms are blocked out in the optical system. Thus the effective complex transmittance of the hologram can be written

$$
t_{H}\left(f_{x}, y\right)=B H^{*}\left(f_{x}, y\right) \exp \left(-j 2 \pi \sin \theta_{H} F f_{x}\right) .
$$

This hologram is placed in plane $P_{2}$ of Fig. 1 .

The image $f(x, y)$ is scanned in a raster format to produce a temporal electronic signal $r(t)$. The raster signal $r(t)$ is related to the 2-D function $f(x, y)$ by the following equation:

$$
r(t)=f\left\{[t-(n-1) T] v_{s}, n \Delta y\right\}
$$

for $n=1$ to $N$, where $v_{s}$ is the scanning velocity of the device (such as a TV camera) that produces the raster signal, $T$ is the duration of each raster line $\left[v_{s} T\right.$ is the size of $f(x, y)$ in the $x$ direction], and $\Delta y$ is the resolution of $f(x, y)$ in the $y$ direction [ $N \Delta y$ is the size of $f(x, y)$ in the $y$ direction]. In Eq. (8) we assume that $f(x, y)=0$ for $x>v_{s} T$ and $x<0$. $r(t)$ is heterodyned to the center frequency $w_{0}$ and applied to the AOD in plane $P_{1}$ of Fig. 1. The modulation $t_{A}(x, t)$ introduced by the AOD on the amplitude of the diffracted light can be found by substituting $a(t)$ by $r(t)$ in Eq. (4):

$$
\begin{aligned}
t_{A}(x, t)= & c_{1} \operatorname{rect}\left(\frac{x-A / 2}{A}\right) r\left(t+\frac{x}{v}-\frac{A}{v}\right) \exp \left(j w_{0} t\right) \\
= & c_{1} \operatorname{rect}\left(\frac{x-A / 2}{A}\right) f\left[\left(t+\frac{x}{v}-\frac{A}{v}-n T+T\right) v_{s}, n \Delta y\right] \\
& \times \exp \left(j w_{0} t\right)
\end{aligned}
$$

We set $A=v_{s} T$, i.e., the aperture of the AOD can accommodate exactly one raster line of $f(x, y)$. For convenience we also set $v_{s}=v$. At time instances $t=n T$ the modulation of the AOD is given by

$$
\begin{aligned}
t_{A}(x, n T)= & c_{1} \operatorname{rect}\left(\frac{x-A / 2}{v T}\right) f\left[\left(n T+\frac{x}{v}-\frac{A}{v}-n T+T\right) v, n \Delta y\right] \\
& \times \exp \left(j w_{0} n T\right) \\
& =c_{1} f(x, n \Delta y) \exp \left(j w_{0} n T\right) .
\end{aligned}
$$

The rect function can be dropped in Eq. (10) since $f(x, y)$ was defined to be nonzero for $0<x<v T$. Thus at times $t=n T$, a single line of the function $f(x, y)$ modulates spatially the light diffracted by the AOD. A pulsed light source is used in the system to illuminate the AOD only at the instances $t=n T$. The temporal modulation of the source can be written as

$$
t_{S}(t)=\operatorname{rect}\left(\frac{t-n T}{\tau}\right)
$$

for $n=1$ to $N$, where $\tau$ is the duration of each light pulse, and the pulse shape has been approximated by a rectangular function. The light diffracted by the AOD is modulated by the product of Eq. (9) and (11). We denote this modulation function by $t_{S A}(x, t)$. For $1 / \tau$ larger than the bandwidth of $r(t), t_{S A}$ can be approximated by

$$
\begin{aligned}
t_{S A}(x, t) & =t_{S}(t) t_{A}(x, t) \\
& =\exp \left(j w_{0} t\right) \operatorname{rect}\left(\frac{t-n T}{\tau}\right) f\left[\left(t+\frac{x}{v}-n T\right) v, n \Delta y\right] \\
& \cong f(x, n \Delta y) \exp \left(j w_{0} t\right) \operatorname{rect}\left(\frac{t-n T}{\tau}\right) .
\end{aligned}
$$

Lens $L_{1}$ in Fig. 1 is used to collimate this light distribution in the vertical direction so it illuminates the hologram in plane $P_{2}$ uniformly in the $y$ direction. Lens $L_{2}$ takes the Fourier transform in the $x$ direction. The amplitude of the light entering plane $P_{2}$ is modulated by

$$
\begin{aligned}
t_{S A}^{\prime}\left(f_{x}, t, n\right) & =\int t_{S A}(x, t) \exp \left(-j 2 \pi f_{x} x\right) d x \\
= & F\left(f_{x}, n \Delta y\right) \exp \left(j w_{0} t\right) \text { rect }\left(\frac{t-n T}{\tau}\right),
\end{aligned}
$$

where

$$
F\left(f_{x}, n \Delta y\right)=\int f(x, n \Delta y) \exp \left(-j 2 \pi f_{x} x\right) d x .
$$

The light immediately after plane $P_{2}$ is modulated by the product of Eqs. (7) and (13). The astigmatic lens system $L_{3}$ images plane $P_{2}$ onto the output plane $P_{3}$ in the $y$ direction, while it performs the Fourier transform in the horizontal $(x)$ direction. The amplitude of the light at plane $P_{3}$ is given by

$$
\begin{aligned}
t_{D}(\hat{x}, \hat{y}, t, n)= & \int t_{S A}^{\prime}\left(f_{x}, t, n\right) t_{H}\left(f_{x}, \hat{y}\right) \exp \left(-j 2 \pi f_{x} \hat{x}^{\prime} d f_{x}\right) \\
= & c_{2} \exp \left(j w_{0} t\right) \operatorname{rect}(t-n T) \int F\left(f_{x}, n \Delta y\right) H^{*}\left(f_{x}, y\right) \\
& \times \exp \left[-j 2 \pi\left(\sin \theta_{H} F+\hat{x}^{\prime}\right) f_{x} d f_{x}\right] \\
= & c_{2} \exp \left(j w_{0} t\right) \operatorname{rect}\left(\frac{t-n T}{\tau}\right) \\
& \times \int f(x, n \Delta y) h(x+\hat{x}, y) d x,
\end{aligned}
$$

where $\hat{x}=\hat{x}^{\prime}+\sin \theta_{H} F$ and $\hat{y}$ are the horizontal and vertical spatial coordinates, respectively, in $P_{3}$, and $c_{2}$ is a constant. The convolution theorem was used to obtain the last form of Eq. (14). The output light distribution is detected interferometrically by the CCD detector array at the output plane to obtain a detected signal proportional to Eq. (14). A reference beam derived from the same source is heterodyned to the center frequency $w_{0}$ of the AOD and made incident on the detector at an angle $\theta_{D}$. The amplitude of the reference beam is described by

$$
t_{R}(\hat{x}, t)=A \exp \left(j 2 \pi \sin \theta_{D} \hat{x} / \lambda\right) \exp \left(j w_{0} t\right) \text { rect }\left(\frac{t-n T}{\tau}\right) .
$$

The signal $I(\hat{x}, y, n)$ that is detected by the CCD is proportional to the time integrated intensity of the sum of Eqs. (14) and (15) 


$$
\begin{aligned}
I(\hat{x}, \hat{y}, n)= & c_{3} \int\left|t_{D}+t_{R}\right|^{2} d t \\
= & \int_{n T-\tau / 2}^{n T+\tau / 2} \mid A \exp \left(j 2 \pi f_{0} x\right) \\
& +\left.\int f(x, n \Delta y) h(x+\hat{x}, \hat{y}) d x\right|^{2} d t \\
= & \left.\tau c_{3}\left|A^{2}+\right| \int f(x, n \Delta y) h(x+\hat{x}, \hat{y}) d x\right|^{2} \\
+ & \left.2 A\left[\int f(x, n \Delta y) h(x+\hat{x}, \hat{y}) d x\right] \cos \left(2 \pi f_{0} x\right)\right\},
\end{aligned}
$$

where $f_{0}=\sin \theta_{D} / \lambda$. The third term in this equation is the 1-D correlation of the $n$th row of $f(x, y)$ with all the rows of $h(x, y)$. The correlation forms on the spatial carrier $f_{0}$. Since both $f$ and $h$ are real functions only the amplitude of the fringe pattern is modulated. In the $\hat{y}$ direction the CCD is operated in the shift-and-add mode described earlier. The charge that is accumulated in the CCD after $N$ light pulses can be found by substituting Eq. (16) into Eq. (5):

$$
\begin{aligned}
& Q(\hat{x}, \hat{y})=\sum_{n=1}^{N} I[\hat{x}, n, \hat{y}+(n-N) \Delta y] \\
& =c_{4}\left\{N A^{2}+\sum_{n=1}^{N}\left|\int f(x, n \Delta y) h[x+\hat{x}, \hat{y}+(n-N) \Delta y] d x\right|^{2}\right. \\
& \left.+2 A\left[\sum_{n=1}^{N} \int f(x, n \Delta y) h(x+\hat{x}, \hat{y}+n \Delta y-N \Delta y) d x\right] \cos \left(2 \pi f_{0} x\right)\right\} .
\end{aligned}
$$

The third term in Eq. (17) forms on a spatial carrier of frequency $f_{0}$. By setting $f_{0}$ equal to or larger than the bandwidth of $f(x, y)$, this term can be separated from the other two baseband terms by electronic filtering after the signal from the CCD is converted to a video signal. The envelope of the carrier in Eq. (17) is recognized to be the 2-D correlation described by Eq. (1) with the integration over the continuous variable $y$ replaced by the summation over the discrete variable $n \Delta y$. The correlation pattern is shifted by the constant $N \Delta y$ in the $\hat{y}$ direction. For a CCD detector with $N$ horizontal rows, this means that each slice of the correlation pattern is formed sequentially at the last row of the device. The fast horizontal CCD shift register transfers each line of the 2-D correlation to the output stage of the device where it can be displayed or processed further electronically.

We shall close this section by discussing how the system of Fig. 1 can be used to correlate complex 2-D functions. We assume that we have the complex function $h(x, y)$ recorded either holographically or in a digital memory. The 1-D Fourier transform of this complex function can then be produced and placed in plane $P_{2}$ of Fig. 1 either optically or by computer generation. The function $f(x, y)$ is represented by two real functions that correspond to its real and imaginary parts. The two corresponding electronic raster signals are used to modulate in quadrature the carrier that is applied to the AOD. From Eq. (4) we conclude that the modulation introduced by the AOD is proportional to the complex signal that is used to modulate in quadrature the carrier. The optical system performs the complex correlation in the $x$ direction. The phase of the 1-D complex correlation is preserved in the phase of the fringe pattern in Eq. (16). Thus the full 2-D complex correlation is formed by performing the addition over $n$ in Eq. (17).

\section{Discussion}

We devote this section to the discussion of the characteristics of the AOD/CCD image correlator. The majority of the potential advantages of this system are consequences of the use of an AOD as the real-time SLM. AODs have been investigated and manufactured for many years. Consequently reliable and relatively inexpensive high quality devices are now commercially available. The simple structure of an AOD relative to most 2-D SLMs allows compact systems to be built with relatively low power requirements and without the need for high voltages. Thus the use of AODs can lead to practically feasible optical image processors in the immediate future. The excellent modulation characteristics of AODs ${ }^{11}$ (high linear dynamic range, spatial uniformity, and low scatter level) allow the input image to be represented in the optical computer with high fidelity, with a corresponding improvement in overall accuracy. AODs can accept data at rates up to several gigahertz. We do not anticipate however, that this capability can be approached by the AOD/CCD image processor in the near future because of limitations in the speed with which the CCD can be read out. The system also requires a device which produces an electronic raster signal corresponding to the input image. In most applications this device can be a TV camera. The current limitations of $\mathrm{CCD}$ and image sensor technologies impose a limit of $\simeq 50-\mathrm{MHz}$ input rate which corresponds to the processing of 200 images, each consisting of $500^{2}$ pixels in 1 sec. The need for an auxiliary image sensor may be construed as a disadvantage of this technique. Ideally it is preferable to have a device which serves simultaneously as the image sensor and the SLM for the optical computer. In many applications, however, 2-D SLMs are not suitable for direct detection of the live scene, and an auxiliary sensor must be used. The output of the auxiliary sensor is then scanned onto the 2-D SLM either electronically or optically. In such cases, the ability of the AOD/CCD system to accept directly the electronic raster signals is an advantage.

The limitations on the performance of the AOD/CCD processor are imposed primarily by the CCD detector. These limitations are not significantly different from the limitations imposed by the detector on any optical image processor. For continuous processing of frames the input data rate cannot exceed the readout rate in the AOD/CCD processor. The speed at which commercially available CCD detector arrays are normally read out is $5-10 \mathrm{MHz}$ (standard video frame rates). Therefore, the processing speed is limited by the detector if one is restricted to using commercially available devices. CCD detectors which are fabricated specifically for optical signal processing applications can be made with several parallel output stages, ${ }^{12}$ which increases the readout speed. For example, a device with 10 parallel readout stages could be read out at 50 $100-\mathrm{MHz}$ rates. In addition, current research in $\mathrm{GaAs}^{13}$ CCDs shows promise of extending the inherent speed 
of these devices to hundreds of megacycles per second. CCD detectors have $\sim 500 \times 500$ pixels, ${ }^{14}$ and devices with over one thousand pixels in each dimension are presently under development. The number of pixels of the CCD in the nonshifting dimension determines in this dimension the space-bandwidth product of the images that can be processed. The number of pixels in the shifting direction determines the space-bandwidth product of the filter in the shifting dimension but does not limit the size of the input scene. In other words, this processor is a sliding window correlator in one dimension. This feature can be useful in applications where the input image is sensed by a linear detector array, and the imaging in the second dimension is accomplished by the relative motion between the detector and the object. The dynamic range, noise, linearity, and spatial nonuniformity of the CCD detector are factors that affect the accuracy with which the processed image can be detected. Commercial CCD cameras have dynamic range in excess of 1000:1 with excellent linearity in this range. ${ }^{14}$ This range can be extended by designing CCDs with large detector elements that can store a larger photogenerated charge and also by cooling the device and using postdetection electronic processing to minimize detector noise. The detector noise is caused by shot noise of the signal and leakage current, feed-through of clock transients, and thermal noise in the amplifiers. ${ }^{14}$ The trade-off between output noise and bandwidth is the major engineering challenge in the design of the AOD/CCD image processor. The portion of the detector dynamic range that is utilized to represent the output signal is proportional to the ratio of the signal to the bias terms in Eq. (17):

$S B R$

$$
2 A \sum_{n=1}^{N} \int f(x, n \Delta y) h(x+\hat{x}, n \Delta y+\hat{y}) d x
$$

$$
N A^{2}+\sum_{n=1}^{N}\left|\int f(x, n \Delta y) h[x+\hat{x}, \hat{y}+(n-N) \Delta y] d x\right|^{2}
$$

For $S B R=1$, half of the dynamic range is utilized. The $S B R$ however can be significantly lower depending on the functions $f$ and $h$. It is thus crucial to maximize the dynamic range of the CCD.

We are considering two types of pulsed source for this system: mode-locked gas lasers ${ }^{7}$ and semiconductor lasers. ${ }^{7}$ Gas lasers can satisfy all the requirements of the system (pulse width, peak power, coherence, sta- bility), but semiconductor lasers are attractive because they are compact, efficient, and relatively inexpensive. The modes in a semiconductor laser, however, are so widely spaced because of the short cavity length that interferometric detection is feasible only with a single-mode laser. Single-mode laser diodes can be fabricated and are even available commercially. However when these diodes are pulsed several modes exhibit gain during the transients. For a well-fabricated diode the transients decay within 1 nsec. ${ }^{15}$ Thus, if a pulse with duration of $5 \mathrm{nsec}$ is used, single-mode operation can be obtained $80 \%$ of the time.

The operation of the CCD detector in the shift-andadd mode has an important advantageous consequence. Since the signal that eventually reaches the output stage of the detector is a weighted sum of the signal detected by all the detector elements in a single column of the CCD array, the variations in the responsivity among the individual pixels and dark current will average out. ${ }^{14}$ In addition, speckle noise which is uncorrelated from pixel to pixel will also average out. Thus the system utilizes coherent light which provides the flexibility of easily synthesizing a filter in the spatial frequency domain but is immune to coherent noise.

\section{References}

1. D. Casasent, Proc. IEEE 65, 143 (1977).

2. W. T. Rhodes, Proc. Soc. Photo-Opt. Instrum. Eng. 180, 143 (1979).

3. D. Psaltis, J. Opt. Soc. Am. 71, 198 (1981).

4. M. O. Hagler, R. J. Marks II, E. L. Kral, J. F. Walkup, and T. F. Krile, Appl. Opt. 19, 4253 (1980).

5. J. C. Chang, IEEE Trans. Sonics Ultrason. SU-23, 2 (1976).

6. K. Bromley, M. Monahan et al., Proc. Soc. Photo-Opt. Instrum. Eng. 118, 118 (1977).

7. A. Yariv, Quantum Electronics (Wiley, New York, 1967).

8. W. T. Rhodes, Proc. IEEE 69, 65 (1981).

9. D. F. Barbe, Proc. IEEE 63, 38 (1975).

10. J. W. Goodman, Introduction to Fourier Optics (McGraw-Hill, New York, 1968).

11. K. Preston, Coherent Optical Computers (McGraw-Hill, New York, 1972), p. 163.

12. G. M. Barsbuk, Proc. IEEE 69, 100 (1981).

13. R. C. Eden and I. Deyhimy, Proc. Soc. Photo-Opt. Instrum. Eng. 214, 39 (1979).

14. J. A. Hall, in Applied Optics and Optical Engineering, Vol. 8, R. R. Shannon and J. C. Wyant, Eds. (Academic, New York, 1980).

15. K. Lau, "Ultra-high Frequency Dynamics of Semiconductor Injection Lasers," Ph.D. Thesis, California Institute of Technology, Pasadena, 1981. 